\title{
7 Where Have All My Languages Gone? Aging and the Changing Multilingual Linguistic Ecology
}

\author{
$\mathrm{Ng}$ Bee Chin and Francesco Cavallaro
}

\section{Introduction}

Effective communication is crucial in the healthcare sector and a growing body of research indicates that the language barriers encountered in healthcare settings may compromise the quality of care (Coren, Filipetto, and Weiss 2009; Schyve 2007; Williams, Weinman, and Dale 1998). This issue is exacerbated when doctors and patients do not share a common language. In the past few decades a considerable amount of research in English-speaking countries has focused on identifying effective practices for improving communication between clinical staff and patients with limited English proficiency (LEP) (Attard et al. 2013; Ngo-Metzger et al. 2007). This is particularly pertinent to Singapore's public healthcare system, which has adopted English as its main working language. However, Singapore displays a different setting to the studies in other countries (the USA, UK, Australia, New Zealand). In these countries, the focus has been on the LEP of new migrants or of migrant populations for whom English is not a mother tongue, and the encounters of these minority groups with the healthcare systems is the usual migrant story of having to integrate or to adapt to a different host culture. For this widely studied group, the issue is of language adaptation in a new land. In Singapore, the language barriers experienced by older adults is a result of the change in the multilingual language ecology resulting in these older adults being displaced as the languages they speak become irrelevant. The cause of this displacement is fundamentally different from the cases that are reported in current literature, but the impact is just as devastating if not more so as it is happening in your home country where you expect to find refuge. This language rupture is not only taking place in the healthcare sector but in the community and at home.

This chapter documents the language problems faced by older adults (OAs) not in a migration context, but in their own home. In Singapore, a significant portion of OAs either do not speak or are not proficient in any of the languages used by the healthcare providers. To this end, we will focus on medical encounters as they are emblematic of the wider social and linguistic barriers experienced by Chinese-speaking OAs in Singapore. 


\section{Limited English Proficiency and Healthcare}

Many studies in English-speaking countries have shown the negative effects of language-discordance between healthcare providers and LEP patients (Regan Freeman 2014; Capps, Rolfe, and Logsdon 2016). Ineffective communication as a result of patients' LEP has been found to worsen health disparity, reduce their access to care, and compromise in quality of care and treatment outcomes (Regan Freeman 2014). Manifestations of this include patients' infrequent visits to healthcare providers, delayed treatments, clinical errors, increased susceptibility to chronic diseases, and health complications (Coren, Filipetto, and Weiss 2009). There are also intangible interactional issues such as patient and provider dissatisfaction, as well as anxieties, misunderstandings, and uncertainties experienced by LEP patients due to the communication problems they face (Coren, Filipetto, and Weiss 2009; Regan Freeman 2014).

An obvious solution is the use of interpreters in medical encounters. "Interpreters" refer to professional interpreters employed by a healthcare institution, or ad hoc, untrained individuals, such as nurses, family members, friends, non-clinical hospital employees, and even strangers from waiting rooms. Ad hoc interpreters tend to lead to significant clinical errors such as omission and substitution of sensitive information, consequently compromising the quality of care. This is due to the fact that the role of interpretation is poorly understood by most untrained individuals (Rosenberg, Seller, and Leanza 2008). Meyer, Pawlack, and Kliche (2010) report that most family interpreters described their role as merely to facilitate understanding rather than to render exactly what was said. Some added that they restricted themselves to interpreting only what they sensed that their family member needed help in or was being misunderstood. Others admitted to not interpreting all interactions between the doctors and patients and deliberately censoring some information as and when they saw fit. Well-meaning, family interpreters can potentially create more misunderstandings or further aggravate existing communicative difficulties.

It is well attested that qualified and trained medical interpreters are significantly less likely to make clinical mistakes than family interpreters, and their use leads to an enhancement in the overall quality of care for LEP patients through a reduction in interpretation errors, increased understanding, with the resultant satisfaction of patients (Karliner et al. 2007; Flores 2005; Betancourt et al. 2012; Arthur et al. 2015). Despite these iron-clad statistics, non-professional interpreters are used worldwide to provide language assistance in many contexts (Rosenberg, Seller, and Leanza 2008; Schenker et al. 2011). As a general practice in the USA, professional interpreters are found mainly in complicated medical processes, whereas those perceived to be less complex often simply involve "getting by" with the use of ad hoc interpreters (Tang et al. 2014; Schenker et al. 2011). 
With the increasing globalization of human movement leading to increased language and cultural diversity, healthcare sectors globally face similar linguistic challenges as they encompass a significant proportion of patients who do not speak the official language(s). This issue is not confined to Singapore or Asia and, indeed, is a pressing concern to cosmopolitan cities all over the world. Despite the fact that these observations are empirically robust, the use of ad hoc interpreters persists in Singapore and in medical systems across the world.

\section{A Linguistic Snapshot of Singapore}

Singapore is multicultural, multilingual, and multireligious. Its population is made up of three major ethnic groups and a significant migrant worker population. The categorization of these three major ethnic groups in many ways obfuscates the teeming diversity in terms of mutually non-intelligible language use within each ethnic group.

Singapore's population has doubled in the last 35 years. This increase is also driven by the size of its transient workforce, which has increased from 200,000 in 1980 to $1,677,391$ or $29.4 \%$ of the total population in 2019 . These are typically manual workers from the region and also includes healthcare providers like nurses and nursing aides from the PRC, India, the Philippines, Myanmar, and Indonesia. These migrant workers, together with the 4,026,209 citizens and permanent residents, bring the current total population to 5,703,600 people (Department of Statistics 2019).

The 4.02 million Singapore citizens and residents are made up of 74.36\% Chinese, $13.43 \%$ Malays, 9.01\% Indians, and 3.21\% of Others (Department of Statistics 2019). There are four official languages, English, Mandarin, Tamil, and Malay, and Malay is also the national language. Other than the four official languages, many other languages are also spoken by Singaporeans. In the Chinese community a number of Chinese vernaculars, such as Hokkien, Cantonese, Teochew, Hakka, and Hainanese (to name the few common ones), are spoken together with Mandarin Chinese. The Indian community consists of speakers of Tamil, Hindi, Punjabi, Bengali, Malayalam, Telugu, and others, and the Malay community also include numbers of Boyanese, Javanese, and Baba Malay (Peranakan) speakers. Most Singaporeans 50 and below are bilingual in English and at least one official languages. Across ethnic groups, the common language is English, which is also sometimes the lingua franca within ethnic groups (Cavallaro, $\mathrm{Ng}$, and Tan 2020). All these communities are experiencing an ongoing language shift toward English, the language used nationwide in business, administration, law, and education. This dramatic social transformation in linguistic ecology has been extensively covered by several researchers (see Cavallaro and $\mathrm{Ng}$ 2014, 2020). A synopsis is presented in the following paragraph. 
The Singapore linguistic landscape has been forcibly shaped by two key language policies: (i) the Bilingual Policy has been emphasizing an Englishplus bilingualism since independence in 1965; and (ii) the Speak Mandarin Campaign (SMC), launched in 1979, promotes Mandarin Chinese over all other Chinese languages. The Bilingual Policy catapulted English as the premium language as it became the medium of instruction in all schools and also the de facto language for all formal contexts, despite the existence of four official languages. Since the launch of the SMC, the census data has been showing a steady increase in the population that uses English as the predominant household language. Economic success has convinced the majority of Singaporeans that a good knowledge of English is the basis for better career opportunities for themselves and their children. The widespread use of English among all ethnic groups and the majority (in numbers) of the ethnically Chinese have elevated English and Mandarin to the status of majority languages, and relegated other Chinese vernaculars, Malay, and Tamil to the rank of minority languages. This period has also seen a dramatic decline in the number of speakers of the Chinese vernaculars, who now largely comprise the OAs in the Chinese community $(\mathrm{Ng}$ and Cavallaro 2021).

A cursory look at the demographic data shows that Singaporeans who are 50 and below (born after independence in 1965) are linguistically well equipped to negotiate the Singapore of today, which requires proficiency in English for virtually all formal domains. Conversely, this same group of Singaporeans have reduced or no capacity in the vernaculars spoken by their parents or grandparents. So, what we have here is a language ecology that has been dramatically transformed over the course of five decades.

Our interest here is with the older Singaporeans. According to the Singapore Population Census, the number of residents aged 65 and above has grown from $6 \%$ in 1990 to $14.4 \%$ of the population in 2019 . In the same period, the median age has increased from 29.8 years to 41.1 (Department of Statistics 2019). The number of OAs are projected to increase to $22.1 \%$ of the population by 2030 and $34 \%$ by 2050 .

As indicated earlier, the OAs in Singapore are in many ways different from those in the UK and the USA where the LEP patients are either new migrants or migrants who have been uprooted from their source culture. This particular group of OAs in Singapore are born and bred in Singapore but are caught in an alien linguistic landscape due to sweeping and dynamic changes in language practices, mainly due to state-driven language policies as well as the forces of globalization.

These changes in language use, attitudes, and repertoire have impacted on OAs in very significant ways. First, as most OAs live with their children and grandchildren, the increase in the use of Mandarin and English in the home domain means that many of these OAs are effectively cut off from communicating directly with their grandchildren (Tan and $\mathrm{Ng} 2010$ ). Second, their lack of proficiency in the country's majority languages means 
that they are unable to interact meaningfully with the wider community. They live in a landscape that is increasingly dominated by the sights and sounds of languages they are not familiar with. They are unable to talk to their grandchildren, use the banks, manage formal transactions on their own, read public signs, and, most important of all, navigate the healthcare system, which is so vital for their well-being. Their language barriers have become a significant handicap as they are increasingly out of sync with the English-dominated new Singapore. Though it is tempting to see this group of OAs as similar to the LEP patients in the literature, they are qualitatively different as for them, the possibility of a home elsewhere does not exist.

For the OAs in Singapore, the inability to access the new language world is very akin to being a stranger in your own home. Hence, instead of referring to this group of OAs as LEP patients, we will refer to them as Linguistically Displaced Older Adults (LDOA). The lives of these LDOAs have transitioned from thriving multilingual and diverse contexts to one where linguistic pragmatism has promoted the rise of some languages to the demise of others at considerable cost to the social well-being of these LDOAs. In the next section, we will focus on medical encounters in the healthcare system as one of the realities of aging is an increased reliance on healthcare providers and medical facilities.

\section{The Healthcare System in Singapore}

An aging population in Singapore, coupled with a projection of the population's greater reliance on the public healthcare system, has brought to the forefront the importance of preparing an equipped workforce and an enhanced healthcare system geared for the future (Cavallaro et al. 2016; Gan 2012). In recent years, there has been a significant and growing proportion of foreign healthcare professionals in Singapore's public healthcare sector due to a shortage of local people joining the sector, as evidenced by the fact that more than $25 \%$ of Singapore's doctors today are foreigners (TST 2015). Coupled with a significant aging population, there is growing dissonance between the language needs of LDOAs and the language used by healthcare professionals pointing to a crucial need for greater consideration of the linguistic challenges faced by these patients. However, the official stance and that of the public hospitals in this aspect has been marked with passivity, albeit with several acknowledgments of a need for greater attention in this area (Neo 2010; Maryam Mokhtar 2014).

The approach on how to tackle these language barriers adopted by the public healthcare sector thus far is to send foreign medical staff (usually the nurses) to language courses in order for them to acquire the necessary (local) languages to converse with patients. A more common practice is to tap on the existing linguistic abilities of local staff (Maryam Mokhtar 2014). Public hospitals, in general, seem to adopt an approach of engaging interpreters only when it comes to foreign languages and not when the language of 


\section{$152 \mathrm{Ng}$ Bee Chin and Francesco Cavallaro}

concern is a local one. An exception is the National University Hospital (NUH), which hired one "dialect" interpreter in 2010, and its plan was to hire more (Neo 2010). To date, however, no other interpreter has been hired. Note that in Singapore the mutually non-intelligible Chinese vernaculars are referred to as "dialects."

To address the needs of the aging population, the Singapore Government set up the Ministerial Committee on Aging (MCA) in 2007, and their vision is "Successful Ageing for Singapore." However, while much has been done in building up the infrastructure to make all spaces more accessible to older residents, very little seems to have been directed at the more intangible aspects of accessibility in terms of the language issues faced by Singaporean LDOAs. The interesting aspect of this is that most of the people of this generation may in fact be very multilingual but are unable to speak or understand English. Their proficiency in Mandarin may also be quite poor (Tan and Ng 2010). Very sadly, most of these LDOAs find themselves surrounded by a world of unfamiliar languages at the final stages of their lives, grappling not only with a myriad of problems associated with aging as well as anxiety and trauma caused by communication barriers.

As indicated earlier, for interpretation needs, local medical institutions simply tap on their own staff who are proficient in the vernaculars (Neo 2010). The Ministry of Health assures the citizens that they roster a good mix of local and foreign healthcare staff at every shift as a measure to reduce the risk of miscommunication. With the advantage of having vernacularspeaking staff, the employment of interpreters for local languages is therefore considered unnecessary by most healthcare institutions.

This chapter will discuss the language issues of LDOAs in the context of medical encounters through a series of semi-structured interviews with LDOAs, nurses, and doctors.

\section{Methodology}

In total, 35 participants (Table 7.1) were interviewed for this study (30 older adults, 5 healthcare providers). The 30 older adults ( 8 males and 22 females) were between the ages of 66 and 83 and were in need of regular

Table 7.1 Participants

\begin{tabular}{lllll}
\hline Males & Females & Main Language & Age & Role \\
\hline 1 & 5 & Mandarin & $68-78$ & Patient \\
3 & 9 & Hokkien & $66-82$ & Patient \\
4 & 8 & Cantonese & $66-83$ & Patient \\
- & 3 & English & $29-71$ & Nurses \\
- & 2 & English & 29 & Doctors \\
\hline
\end{tabular}


medical attention as several have chronic illnesses, such as high blood pressure, prolonged joint issues leading to knee or hip replacement, or diabetes. They were recruited through the network of our research assistants and subsequently by referral sampling as participants then recommended others from their circle of connections. The five healthcare providers were three nurses and two doctors.

The data for the study came from a series of interviews obtained through face-to-face and telephone interviews. Face-to-face semi-structured interviews were conducted with the patient participants and nurses, and telephone interviews were conducted with the doctors.

Prior to each interview, the participants were briefed on the focus of the research, and their verbal informed consent was collected. The interviews with the LDOAs were conducted in Mandarin, Cantonese, and Hokkien, depending on the preferences of the participants. None of the LDOAs were able to speak English, six could speak Mandarin, and the rest only had minimal proficiency in Mandarin Chinese. The other participants reported Hokkien $(\mathrm{n}=12)$ and Cantonese $(\mathrm{n}=12)$ as the language they were most proficient in. The interviews with the nurses and doctors were conducted in English. All participants were interviewed only once and for a time ranging from 15 minutes to 30 minutes. The interviews were audio-recorded, transcribed, and then (those with the LDOAs) translated into English. Upon completion, each participant received a token of appreciation.

Three distinct sets of questions were designed for each target group. The questions to the LDOAs focused on their visits to the larger public hospitals in Singapore and their language proficiency. The questions probed their language needs, for example, whether they needed help when talking to the doctors and how they felt when faced with language barriers. For the nurses and doctors, the questions were aimed at eliciting the strategies they adopted when faced with patients who did not speak English and how they felt about these encounters. To anonymize the data, the interviewees are given a code. The participating LDOAs are referred to according to their gender ( $\mathrm{M}$ or $\mathrm{F}$ ), language $(\mathrm{C}=$ Cantonese, $\mathrm{M}=$ Mandarin, and $\mathrm{H}=$ Hokkien), and age. Therefore, a male Cantonesespeaking participant who was 76 years old would be referred to as: MC76. These identifiers were unique for each participant as no two participants had the same code.

A thematic analysis of the comments by all participants was carried out by the senior members of the research team. However, a consensus on the main themes was arrived at in collaboration with the entire research team. The analysis of the LDOAs focused on communication barriers experienced and their responses and perceptions of difficult medical encounters. The analysis of the interviews of nurses and doctors focused on their perception of their own experience of communication barriers and what resources they drew on. 


\section{$154 \mathrm{Ng}$ Bee Chin and Francesco Cavallaro}

\section{The Findings}

\subsection{Perspective From the Linguistically Displaced Older Adults}

The LDOAs interviewed reported that they were usually accompanied by at least one child during hospital visits or at times came alone. All of them had encountered healthcare professionals with and without Chinese-speaking competency.

The analysis of the transcripts revealed several recurring issues that these elderly patients encountered on their hospital visits:

- Challenges in communicating with doctors,

- Heavy reliance on ad hoc interpreters,

- High dependence on older adults' own initiative for the communication outcome, and

- Passive acceptance of and frustration by the older adults in languagediscordant medical encounters.

\subsubsection{Challenges in Communicating With Doctors}

The respondents who were always accompanied by their children in hospital visits all indicated that the reason they were unable to make these visits alone was due to the English-dominated landscape in public hospitals. By this, they were referring to signage (see the example in Figures 7.1 and 7.2)

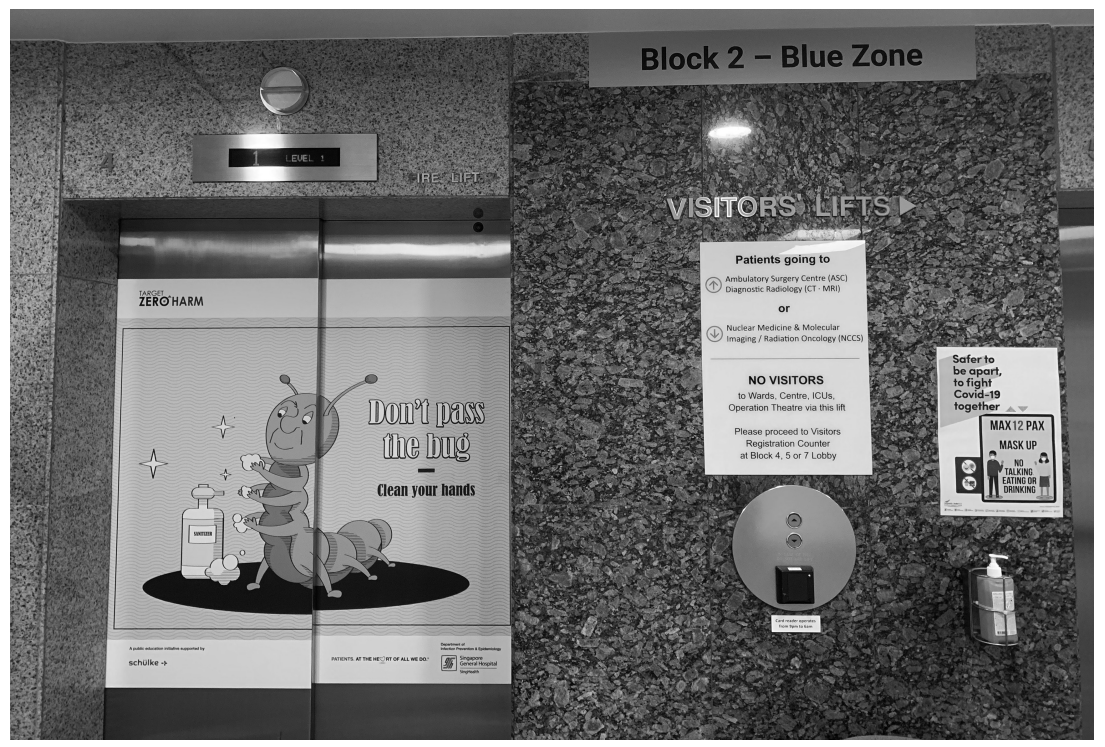

Figure 7.1 Signs outside a lift in a hospital 


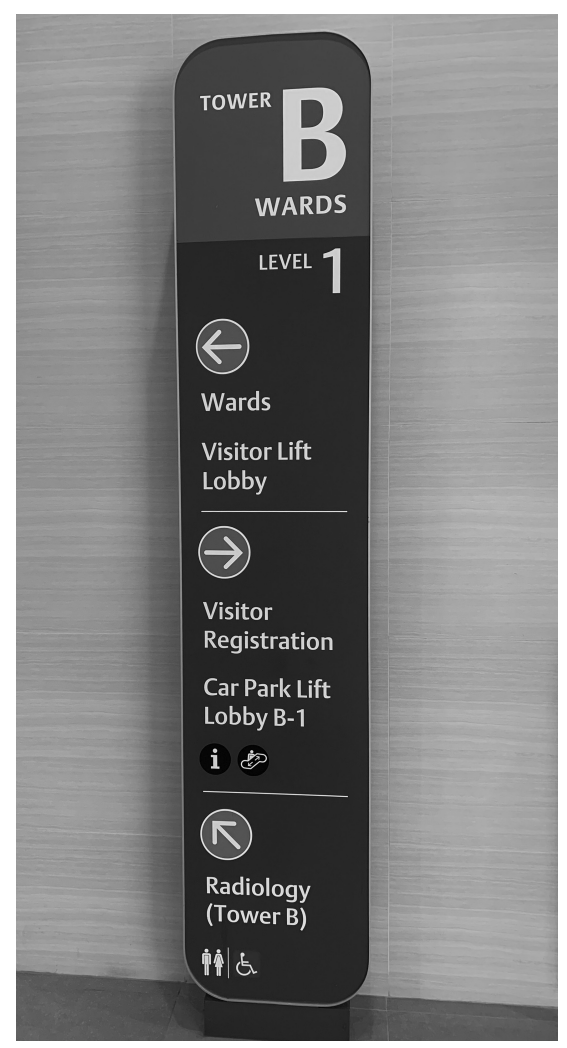

Figure 7.2 Sign at a hospital lobby

in the hospitals as well as simple things such as the announcement of queue numbers and calling out of instructions. All these sensory inputs in hospitals are in English. Many discussed the trips to the hospital as though they were talking about negotiating obstacle courses.

The linguistic landscape of Singapore unambiguously indexes English as the prime language of wider function. Most studies of linguistic landscape document linguistic vitality through the study of signs and their relationship to power and inequality (see Lanza and Woldemariam 2014), but in Singapore, the dominance of English in the landscape is so complete and absolute that the only time it is significant is when it does not dominate. This reflects the greater shift toward a bilingualism in English and an official language. Hence, the English signages in all the hospitals are the first insurmountable barriers for the LDOAs. Without guides, they are unable to negotiate the hospital labyrinth.

The pinnacle of this obstacle course is the language-discordant encounters with their doctors. All respondents pointed out the limitations of the 


\section{$156 \mathrm{Ng}$ Bee Chin and Francesco Cavallaro}

Chinese language competency of doctors, even of those who are ethnically Chinese. The following are some of the responses. (Note that the responses are translated from Chinese languages as none of the LDOAs were able to speak English.)

- "They [the doctors] do not speak Mandarin at all." (FM74)

- "Some Chinese doctors cannot speak Mandarin well ..." (FM78)

- "That day, I consulted a Cantonese doctor, and he was unable to speak Hokkien. He asked if I was able to speak Mandarin. I said if you speak in simple and basic terms, I will be able to understand. But if you get too complicated then I will not be able to understand." (MH81)

- "I can understand simple terms." (FH82)

Anything complicated posed a challenge for some of these LDOAs. With the limited Chinese-speaking competencies of the doctors, the level of understanding by the patients is consequently compromised, as seen in the responses below:

- "They [the doctors] speak in English; I do not understand." (FM77)

- "I am able to understand the Chinese doctors better, however sometimes they also cannot speak Mandarin well, and they have to ask the nurses to interpret." (FM78)

The LDOAs repeatedly emphasized their doctors' usage of English or limited use of Mandarin, and their consequent inability to understand or be engaged in a conversation without the aid of ad hoc interpreters. This is further illustrated on in the following section.

\subsubsection{Reliance on Ad Hoc Interpreters}

The responses of the elderly patients reveal a reliance on ad hoc interpreters, especially on their children and nurses. A common thread across responses given is that as doctors tend to be assisted by nurses, the LDOAs took for granted that the nurses' job scope includes being interpreters.

- "He [the doctor] will ask a nurse to come into the room ... the nurse who can speak Hokkien will interpret for me." (FH72)

- "When they [the doctors] speak in English I am unable to understand, unless there is an interpreter [a nurse] next to the doctor." (FM77)

- "If I don't understand, I look for nurses and ask them." (FM77)

- "If the doctors cannot speak Mandarin, they will ask others such as 'missy' (nurses), administrative staff, or people who do chores in the hospitals for help" (FM70)

The nurses functioned as mediators between patients and doctors and they were also the hospital personnel whom patients sought out when faced with 
difficulties in understanding or any other uncertainties. Some of the LDOAs saw resorting to nurses as the only option as seen below:

- "As we cannot speak in English, there is no choice but to ask someone in to help us understand better. If we meet a Hokkien nurse, we can at least tell her that things are like this and that and she will understand us." (FH72)

- "There's no choice even if it is troublesome. We have to ask around if we do not understand." (FM78)

With a quarter of doctors in Singapore being foreign and the high number of non-Singaporean nurses and other healthcare workers, as well as the number of younger Singaporeans who may not know local languages or even Mandarin, the nurses who are able to provide this service is decreasing. Indeed, some LDOAs reported that doctors have resorted to asking clerical or administrative staff in the office for assistance when no nurses with the language ability could be found. In the following excerpt, FM77 reported that a fellow patient in the ward pitched in as an ad hoc interpreter.

- “There was once my neighbour's (the patient in the bed next to her) visitor had to help to translate what the healthcare professionals were saying to me as Hospital A has many foreign doctors and nurses who can only speak English." (FM77)

\subsubsection{Family Members as Interpreters}

It was also common for these LDOAs to be accompanied by family members who would speak on their behalf during their healthcare visits.

- "My daughter will speak for me. There is no need for me to speak." (FC72)

- "My son and the doctor would speak in English, a language that I do not understand. I will then ask my son about what the doctor say, and he will explain to me." (FC72)

- "I use Hokkien and she (my daughter) will interpret it into English for the doctor." (FH72)

The responses above, once again underscore the notion of dependence - in this case, on family members. The following is an example of an LDOA who went for the first time to the hospital without her children as she was not anticipating any significant language problem. Her coping mechanism was to remain silent despite completely not understanding what her doctor was saying:

- "There was once I went for a hospital visit alone without any company, I did not understand anything and chose to stay silent and just leave it. 
I simply took my medicine, paid the bill, and went home. Hence, for subsequent visits, my children have to accompany me." (FM74)

In another case, despite having an underlying desire for autonomy, another LDOA allowed her son to speak on her behalf, because she felt that her son was more likely to take time to explain things to her, unlike a doctor. However, FH79 still clearly preferred hearing the information directly herself instead of from her son.

- "It will be much clearer when we hear it for ourselves. It may not be that clear if my son listens on my behalf. My son might forget some things that the doctor says. When we hear it ourselves, we will put in more effort to remember what is being said." (FH79)

Using family members as interpreters is low cost and convenient, but this strategy is not without its problems. This strategy deprives the LDOAs of a sense of autonomy and it also deprives them of the possibility to be their own health advocate. This disempowerment has other negative knockon effects. The case of FM74 who pretended to understand the consultation is potentially dangerous and posed a serious risk to her well-being. Medication compliance for older adults who have multiple chronic conditions can be very complicated and any error in following instructions due to language barriers have been found to have very disastrous ramifications (Suppiah et al. 2020).

\subsubsection{Frustration and Passive Acceptance}

Overall, the LDOA participants indicated that, despite the frustration of not having a common language, they still trusted and complied with the doctor's instructions. For example, MM68 felt that it was not important to understand everything his doctor said.

- "Just catching the approximate meaning is sufficient, I do not need to think so much about it." (MM68)

From a medical viewpoint, this passive acceptance of language barriers can be a problem. As pointed out earlier, the consequent inability of LDOAs to make informed health decisions can be detrimental if they have limited health literacy and are unable to sufficiently comprehend health information in language-discordant medical encounters (Capps, Rolfe, and Logsdon 2016).

Only one LDOA in our group was assertive enough to ensure she understood what the healthcare professionals were saying by insisting on repetitions and clarifications. Several on the other hand, expressed their frustration, especially when recounting their hospitalization experiences: 
- "Whenever an urgent need comes up and no one can communicate in Mandarin, it becomes frustrating for me as I have no choice but to use gestures." (MM68)

- "When they (the hospital) are shorthanded and I am really left without choice, especially during night shifts, I have to attempt to use broken Chinese or extremely basic English words to get my message across." (FM78)

The frustration and sense of desperation is evident in the above excerpts and such emotional distress and anxiety was common in the account of their healthcare encounters. The next sections provide an account of the views of some health professionals.

\subsection{Perspective From the Nurses}

The interviews were conducted in English with three nurses who worked in public hospitals and interacted with a significant number of LDOAs in the course of their careers. The first nurse interviewed, Eve (all names are pseudonyms), was 29 years old and had two years of nursing experience in public hospitals. She was fluent in English, Mandarin, and Cantonese, and she was able to speak basic Malay and Hokkien. The second nurse, Dorcas, was 71 years old and had 53 years of nursing experience in public hospitals and mobile clinics. She was fluent in English, Mandarin, Hokkien, Teochew, and Cantonese, and some basic Malay. The last nurse, Lucy, was 69 years old and had 50 years of experience as a nurse in public hospitals and polyclinics. She was fluent in English, Mandarin, Hokkien, and Cantonese, with basic Malay, Teochew, and Hakka. The recurring themes these nurses encounter at work in public hospitals are as follows:

- A focus on "getting by" medical encounters each day,

- Their own reliance on ad hoc interpreters and their role as ad hoc interpreters,

- Common concern of time wastage and distraction from core responsibilities.

\subsubsection{Focus on "Getting By" in Medical Encounters}

The responses of the nurses reveal that simply being able to "get by" is of primary concern to them during medical encounters. Tang et al. (2014) defined "getting by" as knowing that the practice of using an ad hoc interpreter is not ideal but doing it anyway as it was the only solution. "Getting by" also involves omitting information if no interpreters were available. Basically, "getting by" means you just make do with whatever resources you have. 


\section{$160 \mathrm{Ng}$ Bee Chin and Francesco Cavallaro}

- "If we [the nurses] cannot find a translator, we will call the patients' family members. If we cannot get through, then there is no choice (an attempt to communicate stops)." (Eve, 29)

- "Sometimes when we [the nurses] are left without choice because we really cannot find the people who can speak the required language, we have to make do with limited communication and understanding." (Lucy, 69)

\subsubsection{Reliance on Ad Hoc Interpreters}

It is clear from the three nurses that the use of $a d$ hoc interpreters was prevalent in medical encounters as gathered from the nurses' responses:

- "During interactions with my patients, I will ask my colleagues or the patients' children to translate for me if I do not know how to speak the language well enough." (Lucy, 69)

- "If I face difficulty communicating with patients, I rely on existing staff, the family members at their bedside or their next-door patients to translate for me." (Eve, 29)

Similar to the LDOAs in this study, these nurses also relied extensively on ad hoc interpreters, which consisted of their colleagues and the LDOA's children. When these groups of people were unavailable, the nurses sought help from other patients or other hospital staff, or anyone else who was happened to be there.

\subsubsection{Nurses' Role as Ad Hoc Interpreters}

As indicated earlier, LDOAs tended to see nurses as one of the main groups of ad hoc interpreters they can rely on. These nurses felt the pressure of this reliance on a day to day basis.

- "I can be called to be an interpreter a few times a day." (Dorcas, 71)

- "The frequency of me being an ad hoc interpreter is about 20 to $30 \%$." (Eve, 29)

Part of making do means that the nurses often had to interpret outside their comfort zone:

- "Sometimes when I communicate in languages that I am weak in, such as Malay, I will have trouble expressing myself or understanding the patients." (Lucy, 69)

- "For me, communicating is not much of an issue but sometimes it gets challenging when I have to speak in Malay." (Dorcas, 71) 
Their struggle with the responsibility of having to interpret in languages they were not so familiar with was a source of stress and anxiety. Dorcas expressed her guilt, worry, and struggle as she grappled with communicating in her less fluent languages. Eve acknowledged her limitations in medical encounters of greater complexity as she struggled to relay the message accurately, especially when they involved the use of medical terminologies:

- "Usually, I have no problem communicating the basic things in different languages even with my very limited Hokkien and Malay language skills. However, once the subject matter gets more complicated, for example, when I have to use medical terms in a patients' language, I face problems going in-depth even if I am proficient in the language. This can get very stressful" (Eve, 29)

\subsubsection{Concerns Over Time Wastage and Distraction From Core Responsibilities}

The element of time lost or wasted was one of the foremost concerns for nurses as they repeatedly raised time wastage as a negative yet inevitable implication of language-discordant medical encounters:

- "If I cannot communicate with the patients, I may use gestures. However, it takes a longer time." (Lucy, 69)

- "At times, there will be a lot of impatience, frustration, and unhappiness from both patients and doctors when they have to search and wait for an ad hoc interpreter" (Dorcas, 71)

- "If I am doing something, I have to ask them (people who need ad hoc interpreters) to wait." (Lucy, 69)

They were aware of how efforts to mitigate or circumvent language barriers such as the use of gestures, or the searching and waiting for the availability of ad hoc interpreters often resulted in more time wasted which consequentially created frustration and unhappiness in both patients and healthcare professionals. A common thread in their responses was the negative distraction that ad hoc interpretation brought to their work:

- "It takes time from my own ward." (Lucy, 69)

- "When you come back to your own room (ward) after interpreting for others, it is in a mess. There is no choice but to neglect my own room when I am called to be an interpreter. There is also no one to help to take over my room as everyone else is too busy." (Dorcas, 71)

- "Being an ad hoc interpreter causes me to neglect my own patients." (Eve, 29) 


\section{$162 \mathrm{Ng}$ Bee Chin and Francesco Cavallaro}

They all saw this distraction as compromising of the quality of care received by all patients - not only the LDOAs needing language assistance.

\subsection{Perspective From the Doctors}

The two doctors interviewed worked in public hospitals and have encountered a significant number of LDOAs in their professional lives. Both of the doctors interviewed were Chinese Singaporean females (29 years old) and both had four years of experience working in public hospitals. The first doctor, Faith, was fluent in English and Mandarin, and was able to converse in minimal Malay and Hokkien. The second doctor, Rachel, was likewise fluent in English and Mandarin, with minimal knowledge in Malay and a variety of dialects. Two recurring themes have been identified from the responses of these doctors:

- A similar focus on "getting by."

- An acceptance of the "getting by" philosophy.

\subsubsection{Focus on "Getting By" in Medical Encounters}

Like the nurses, the doctors' focus on "getting by" is clear.

- "Professional interpreters are only used in language encounters which involve rare languages like Arabic or Japanese. For the usual national languages, I will just ask for help from existing staff." (Rachel, 29)

- "I will get patients' family members or healthcare workers to help with translation when I am faced with language barriers in communication." (Faith, 29)

The responses confirmed that the use of ad hoc interpreters, most commonly healthcare staff and LDOAs' family members, was one of the primary ways through which doctors coped with language barriers. Although, there was an awareness that this reliance on ad hoc interpreters had its limitations:

- "It is hard to tell how accurate ad hoc interpreters are, as I do not understand the language myself. I would assume that if the interpreters are nurses or other healthcare professionals, interpretation would be better and more accurate, whereas if I ask the administrative staff for help, I think that they also struggle with interpretation." (Faith, 29)

Faith admitted that she was unable to ascertain the effectiveness or accuracy of ad hoc interpretations. The fact that ad hoc interpreters are ultimately untrained, coupled with a doctors' inability to gauge the effectiveness or outcome of communication, opens the possibility for undetected erroneous 
or incomplete interpretation. Other examples of this "getting by" strategy are shown in the following excerpts.

- "I can understand and speak a little dialect [Chinese vernaculars], so I can still get by in these encounters." (Faith, 29)

- "Occasionally, when I face difficulty in expressing myself, I will add in some broken English to try to get my message across." (Rachel, 29)

Faith acknowledged the difficulty when there was a need to use medical terminologies or when she had to talk about more complicated issues. The doctors' response below highlights the potential problem of ineffective communication posed by the "getting by" approach:

- "Whenever I try to explain things in Mandarin or dialect, especially when it comes to medical terminologies or more complicated issues, language barrier becomes significant." (Faith, 29)

- "Many of my colleagues and I cannot speak Mandarin well, so in communicating with patients some meaning is inevitably lost along the way. When it comes to medical terms, this [loss] becomes quite significant." (Rachel, 29)

\subsubsection{An Acceptance of the "Getting By" Philosophy}

Though the doctors were cognizant of the problems and potential dangers of this "getting by" approach, there is a sense that they perceived this to be an accepted norm and hence, not something that is likely to change.

- "[In utilizing ad hoc interpreters] We have been able to get by with no serious mistakes; professional interpreters are not crucial, but will be belpful." (Rachel, 29)

- "Language barrier compromises the quality of care received by patients to a little extent as it does not really affect their health or recovery; it is just that these patients may not understand as much as they could have, but it is not anything dangerous or unsafe." (Rachel, 29)

Basically, though the circumstances deviated from the ideal treatment, they were considered acceptable and, according to the doctors, did not compromise care in any way.

It is interesting that both doctors' trust in the safe functioning of the "getting by" approach is not in tune with the research on the importance of sound communication and patient care. In a review of 36 papers on this topic, Flores (2005) presents a correlation between the lack of professional interpretation with poorer patient satisfaction, quality of care, and health outcomes. This is not only measured in terms of better overall health outcomes for the patients, but also reflected in a significant reduction of cost 


\section{$164 \mathrm{Ng}$ Bee Chin and Francesco Cavallaro}

to the healthcare system. Jacobs et al. (2004) report that when patients are provided with professional interpretation services, they are more likely to make proactive health choices resulting in healthcare savings, which are nine times the cost of providing a professional interpreter. A more recent review by Brandl, Schreiter, and Schouler-Ocak (2020) continues to support the call for more widespread use of professional interpreters, indicating that it benefits both the patients and the healthcare systems. The sense of complacency shown by the doctors in our study may in itself indicate a dangerous complacency which should be a public health concern. In short, "getting by" is just not "getting it."

\section{Discussion}

From the responses of all three groups of respondents it is clear that the patients and health professionals experience language-discordant encounters and often had to face the repercussions of this problem. These challenges faced, and the consequent compromise in their communications, inevitably led to frustrations experienced by the LDOAs. Similarly, nurses - who were often called away from their core duties to be an ad hoc interpreters - also recognized the problems in such encounters in terms of poor communication, inefficiency, and compromised patient care.

The doctors, on the other hand, have accepted the "getting by" philosophy all too readily. This has culminated in a mindset that sees the use of ad hoc interpreters as a benign phenomenon and, therefore, the status quo. While it is true that there have been no overt disasters that can be attributed to the use of $a d$ hoc interpreters, there are hidden costs to the health system, such as patients' infrequent visits to healthcare providers, delayed treatments, clinical errors, an increased susceptibility to chronic diseases, and health complications (Capps, Rolfe, and Logsdon 2016; Coren, Filipetto, and Weiss 2009).

To summarize, there are three key problems associated with the current practice. First, these ad hoc interpreters may possess a varying degree of proficiency in the vernacular or Mandarin when attempting to interpret. Second, leaning on nurses as a source for interpretation can overburden the nurse, and this interruption of their core duties has been found to be a stressor that distracts nurses and interferes with their workflow. This has other implications as well, as Duchêne puts it $(2011,102)$, the language skills of these semi-visible workers "are not considered worthy of recognition ... they are inventoried and can be called upon - in this sense, they are exploitable - when the institution needs them to successfully complete its work." He goes on to argue that the company itself, in this case the hospital, is the only beneficiary of this mode of communication. Third, the interpretation done by ad hoc interpreters may not be fully representative of the LDOA patients' actual health condition, especially as they are not trained 
medical interpreters, and they may not know the correct medical terms. This results in situations where medical information may be lost in the process of relaying the doctor's message.

\section{Conclusion}

This chapter provides an account of the language barriers experienced by LDOAs in medical encounters in Singapore. The inadequate existing measures exposes our older adults to potential risks of clinical errors and of subjecting them to a compromised quality of care. The medical encounters are emblematic of the other daily linguistic challenges they have to face. On an individual level, this language displacement has resulted in the disruption in intergenerational communication due to differing language repertoires between the younger and older generations and contributes to a keen sense of isolation felt by these older adults. Aging on its own is not without its challenges, but for this group of older adults who are displaced, the challenges are multi-fold. They have been deprived of their familiar sights and sounds, but as a society, at least in the very important healthcare settings, we could provide them with the means for them to feel cherished and empowered. Such means are readily available in the community in the form of well-trained multilingual facilitators. From the point of view of linguistic justice, these LDOAs have been marginalized by a system that privileges one language over another. In the schema of linguistic justice articulated by (Van Parijs 2002, 2011), the evaluation of linguistic justice is always synchronic and does not take into account historical change and evolution and the casualties in the process. Cross-border English hegemony and its initial inequality is seen as both inevitable and unavoidable. In the case of the LDOAs in Singapore, the diachronic within-border experience of the different forms of multilingualism is certainly a key factor in their experience of linguistic anomie. Younger generations born into the Singaporean Englishdominant economy may have bought into it and, therefore, minimized the effect of the linguistic injustice on them. However, for this aging group of LDOAs, the spread of English within their community has definitely given rise to unequal multilingualism within their national borders and within their lifetime. We believe that the invisible voices of these LDOAs are precisely what Lanza and Lomeu Gomes (2020) identified as one of the multilingual practices that are not addressed in the Global North, which so far has been very engaged in migration and transnationalism studies. Singapore joins the rank of 33 other nations to be "superaged" in ten years where $22 \%$ of the population will be over 65 . This number is set to increase to $34 \%$ in 2050. Hopefully, it will have a multilingual workforce to adequately cater to this burgeoning population as well as that of the more than 1 million migrant workers. 


\section{$166 \mathrm{Ng}$ Bee Chin and Francesco Cavallaro}

\section{References}

Arthur, K., R. Mangione-Smith, H. Meischke, C. Zhou, B. Strelitz, M. Garcia, and J. Brown. 2015. "Impact of English Proficiency on Care Experiences in a Pediatric Emergency Department." Academic Pediatrics 15 (2): 218-224.

Attard, M., A. McArthur, D. Riitano, E. Aromataris, C. Bollen, and A. Pearson. 2013. "Improving Communication between Health-care Professionals and Patients with Limited English Proficiency in the General Practice Setting." Australian Journal of Primary Health 21 (1): 96-101.

Betancourt, J.R., M.R. Renfrew, A.R. Green, L. Lopez, and M. Wasserman. 2012. Improving Patient Safety Systems for Patients with Limited English. Agency for Healthcare Research and Quality. Cambridge, MA: Agency for Healthcare Research and Quality.

Brandl, E.J., S. Schreiter, and M. Schouler-Ocak. 2020. “Are Trained Medical Interpreters Worth the Cost? A Review of the Current Literature on Cost and Cost-Effectiveness." Journal of Immigrant and Minority Health 22 (1): 175-181.

Capps, J., S. Rolfe, and C. Logsdon. 2016. "Limited English Proficiency: Impact on Health Literacy and Health Disparity." The Kentucky Nurse 64 (1): 13-14.

Cavallaro, F. and B.C. Ng. 2014. "Language in Singapore: From Multilingualism to English Plus." In Challenging the Monolingual Mindset, edited by Y. Slaughter and J. Hajek, 33-48. Bristol, UK: Multilingual Matters.

Cavallaro, F. and B.C. Ng. 2020. "Chapter 8: Multilingualism and Multiculturalism in Singapore." In Multilingual Global Cities: Singapore, Hong Kong and Dubai, edited by P. Siemund and J.R.E. Leimgruber. New York, USA: Routledge.

Cavallaro, F., B.C. Ng, and Y.-Y. Tan. 2020. "Singapore English.” In Handbook of Asian Englishes, edited by Kingsley Bolton, Weerner Botha, and Andy Kirkpatrick, 419-448. New Jersey, USA: Blackwell-Wiley.

Cavallaro, F., M.F. Seilhamer, Y.T.F. Chee, and B.C. Ng. 2016. "Overaccommodation in a Singapore Eldercare Facility." Journal of Multilingual and Multicultural Development 37 (8): 817-831.

Coren, J., F. Filipetto, and L. Weiss. 2009. "Eliminating Barriers for Patients with Limited English Proficiency." The Journal of The American Osteopathic Association 109 (12): 634-640.

Department of Statistics. 2019. Population Trends 2019. Singapore: Department of Statistics, Ministry of Trade \& Industry.

Duchêne, A. 2011. "Neoliberalism, Social Inequalities, and Multilingualism: The Exploitation of Linguistic Resources and Speakers." Langage et société 136 (2): 81-108. doi: 10.3917/ls.136.0081.

Flores, G. 2005. "The Impact of Medical Interpreter Services on the Quality of Health Care: A Systematic Review." Medical Care Research and Review 62 (3): 255-299.

Gan, K.Y. 2012. "Speech by Mr Gan Kim Yong, Minister for Health at The Groundbreaking of the Lee Kong Chian School of Medicine's Novena Campus." https://www.asiaone.com/health/coming-more-doctors-2020.

Jacobs, E.A., D.S. Shepard, J.A. Suaya, and E.-L. Stone. 2004. "Overcoming Language Barriers in Health Care: Costs and Benefits of Interpreter Services." American Journal of Public Health 94 (5): 866-869.

Karliner, L., E. Jacobs, A. Chen, and S. Mutha. 2007. "Do Professional Interpreters Improve Clinical Care for Patients with Limited English Proficiency? A Systematic Review of the Literature." Health Services Research 42 (2): 727-754. 
Lanza, E., and R. Lomeu Gomes. 2020. "Family Language Policy: Foundations, Theoretical Perspectives and Critical Approaches." In Handbook of Home Language Maintenance and Development: Social and Affective Factors, edited by A.C. Schalley and S.A. Eisenchlas, 153-173. Berlin: De Gruyter Mouton.

Lanza, E. and H. Woldemariam. 2014. "Indexing Modernity: English and Branding in the Linguistic Landscape of Addis Ababa." International Journal of Bilingualism 18 (5): 491-546.

Mokhtar, Maryam. 2014. "Parliament: More Than 2,000 Foreign Nurses Went for Language Courses in last 5 Years.” The Straits Times. https://www.asiaone. $\mathrm{com} /$ health/parliament-more-2000-foreign-nurses-went-language-courses-last-5years.

Meyer, B., B. Pawlack, and O. Kliche. 2010. "Family Interpreters in Hospitals: Good Reasons for Bad Practices.” mediAzioni 10 (Special Issue 2010): 297-342.

Neo, C.C. 2010. "Communicating with Patients: How Do You Say 'Healthcare' in Hokkien? Today Newspaper Singapore.” Today. https://eresources.nlb.gov.sg/ newspapers/digitised/issue/today20100301-3.

Ng, B.C., and F. Cavallaro. 2021. "The Case of Mandarin Chinese in Singapore.” In Multilingual Singapore: Legacy, Status, and Prospects, edited by Ritu Jain. New York, USA: Routledge.

Ngo-Metzger, Q., D. Sorkin, R. Phillips, S. Greenfield, M. Massagli, B. Clarridge, and S. Kaplan. 2007. "Providing High-Quality Care for Limited English Proficient Patients: The Importance of Language Concordance and Interpreter Use.” Journal of General Internal Medicine 22 (2 Supplement): 324-330.

Regan Freeman, R. 2014. "Primary Care for Limited English-speaking Patients and Parents." Journal of The American Association of Nurse Practitioners 27 (3): 167-176.

Rosenberg, E., R. Seller, and Y. Leanza. 2008. “Through Interpreters' Eyes: Comparing Roles of Professional and Family Interpreters.” Patient Education and Counseling 70 (1): 87-93.

Schenker, Y., E. Pérez-Stable, D. Nickleach, and L. Karliner. 2011. "Patterns of Interpreter Use for Hospitalized Patients with Limited English Proficiency." Journal of General Internal Medicine 26 (7): 712-717.

Schyve, P. 2007. "Language Differences as a Barrier to Quality and Safety in Health Care: The Joint Commission Perspective.” Journal of General Internal Medicine 22 (2 Supplement): 360-361.

Suppiah, S., Y.W. Tan, G.H.-L. Cheng, W.E. Tang, and R. Malhotra. 2020. "Mediators of the Association of Limited English Health Literacy with Medication Non-adherence among Singaporean Elderly." Proceedings of Singapore Healthcare 29 (1): 25-32. doi:10.1177/2010105819899126.

Tan, S. and B.C. Ng. 2010. "Three Generations Under One Roof: A Study of the Influence of the Presence of Grandparents on Language Shift, Identity and Attitudes." TRANEL Travaux neuchâtelois de linguistique 52: 69-92.

Tang, A., J. Kruger, J. Quan, and A. Fernandez. 2014. "From Admission to Discharge: Patterns of Interpreter Use among Resident Physicians Caring for Hospitalized Patients with Limited English Proficiency." Journal of Health Care for the Poor and Underserved 25 (4): 1784-1798.

TST. 2015. "Number of Foreign Doctors Rising in Singapore Public Hospitals and Polyclinics." The Straits Times. http://www.straitstimes.com/singapore/health/ number-of-foreign-doctors-rising-in-singapore-public-hospitals-and-polyclinics. 


\section{$168 \mathrm{Ng}$ Bee Chin and Francesco Cavallaro}

Van Parijs, P. 2002. "Linguistic Justice.” Politics, Philosophy \& Economics 1 (1): 59-74.

Van Parijs, P. 2011. Linguistic Justice for Europe and for the World. Oxford: Oxford University Press.

Williams, S., J. Weinman, and J. Dale. 1998. "Doctor-patient Communication and Patient Satisfaction: A Review.” Family Practice 15 (5): 480-492. 